\title{
Sף УПРАВЛЕНИЕ БЕЗОПАСНОСТЬЮ
}

В.Л. Шульц, В.В. Кульба, А.Б. Шелков, И.В.Чернов

\section{МЕТОДЫ И МЕХАНИЗМЫ ПЛАНИРОВАНИЯ И УПРАВЛЕНИЯ В УСЛОВИЯХ ЧРЕЗВЫЧАЙНЫХ СИТУАЦИЙ}

\begin{abstract}
Аннотация: Рассмотрены основные особенности процессов планирования и управления в условиях чрезвычайных ситуачия (ЧС). Поставлена и решена задача оптимального планирования превентивных мер по предупреждению ЧС в регионе, заключающаяся в выборе комплекса мероприятий для предотвращения (исключения причин возникновения и снижения тяжести последствий развития) чрезвычайных событий. В качестве критериев эффективности предложено использовать размер возможных людских потерь, объем нанесенного ЧС материального ущзерба или размер суммарных затрат на ввод в действие сил и средств при реализаџии превентивных мер, направленных на предупрељдение ЧС. Поставленная задача сведена $\kappa$ задаче иелочисленного (булевого) программирования и может быть решена стандартными методами.Приведены формализованные методы решения задач оперативного планирования проиессов предупреждения и ликвидации последствий ЧС различного типа. Рассмотрен комплекс проблем повышения эффективности использования временных, ресурсных, структурнотехнологических, природно-климатических и эколого-физиологических видов резервов при решении задач оперативного управления в условиях ЧС.Рассмотрена задача минимизации максимального времени проведения комплекса аварийно-спасательных и других неотложных работ на объектах управления при ограничениях на величину конечных потерь, ущербов и затрат на ввод в действие необходимых сил и средств. Результаты решения поставленной задачи позволяют региональным органам управления получить опорный превентивный план действий в условиях наступления и развития ЧС в соответствии со сформированным региональным сценарием и поставленным ему в соответствие графом причинно-следственных связей чрезвычайных событий. Поставленная задача относится к классу задач иелочисленного программирования.Изложены результаты анализа эффективности использования сценарного подхода при решении задач планирования и управлении в условиях ЧС. Показано, что широкое использование нормативной базь в прочессе сценарного исследования обеспечивает возможность комплексного подхода к решению задач управления предупреждением и ликвидацией последствий ЧС, позволяющего одновременно рассматривать взаимосвязанные, но приничпиально различные по своей природе явления и проиессы. Это обеспечивает возможность достаточно эффективного учета в единой сценарной модели факторов и угроз террористической, пожарной, радиационной, химической, энергетической, экологической и т.д. безопасности.
\end{abstract}

Ключевые слова: Безопасность, планирование, управление, сценарный анализ, имитационная модель, прогноз, угроза, чрезвычайная ситуащия, ущчерб, резерв 


\section{Введение}

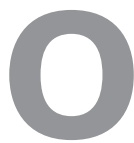
сновные особенности процессов управления в условиях техногенных аварий и катастроф состоят в том, что чрезвычайная ситуация (ЧС) возникает и развивается неожиданно, внезапно. С момента ее возникновения перед системой организационного управления встают задачи, принципиально не свойственные стационарному режиму работы. При этом, что особенно важно, контрмеры (мероприятия по противодей- ствию развитию ЧС и ликвидации ее последствий) должны приниматься незамедлительно и быть максимально эффективными. Одновременно с этим перед системой управления возникают принципиально новые задачи, усложняющиеся мощным потоком поступающей исходной информации, которую требуется изучить и оперативно проанализировать.

Анализ процессов управления в условиях ЧС позволил выделить ряд их особенностей по сравнению с режимом повседневной деятельности, наиболее типичные из них приведены в табл. $1^{1}$.

Таблий 1.

Сравнительные характеристики процессов управления

\begin{tabular}{|c|c|}
\hline $\begin{array}{c}\text { Режим повседневной } \\
\text { деятельности }\end{array}$ & Управление в условиях ЧС \\
\hline Постоянный режим функционирования. & Различные режимы функционирования. \\
\hline $\begin{array}{c}\text { Жесткая структура и четкое распределение } \\
\text { функций на длительный период. }\end{array}$ & $\begin{array}{c}\text { Отсутствие жесткой структуры и четкого } \\
\text { распределения функций на длительный период, } \\
\text { гибкость, агрессивность. }\end{array}$ \\
\hline Узкая функциональная направленность. & $\begin{array}{l}\text { Широкая и частично непредсказуемая область } \\
\text { действия. }\end{array}$ \\
\hline Моноструктура. & Полиструктуры. \\
\hline Регламентированные информационные потоки. & $\begin{array}{c}\text { Зависимость информационных потоков от } \\
\text { складывающейся ситуации. }\end{array}$ \\
\hline Точная информация. & Недостоверная информация. \\
\hline Избыточная информация. & Недостаточная информация. \\
\hline Невысокий темп изменений. & Высокий темп изменений. \\
\hline Предсказуемость ситуаций. & $\begin{array}{l}\text { Непредсказуемость ситуации; ориентация на } \\
\text { прошлый опыт, как правило, не имеет смысла. }\end{array}$ \\
\hline $\begin{array}{c}\text { Принцип единства полномочий и } \\
\text { ответственности. }\end{array}$ & $\begin{array}{c}\text { Сочетание принципов единоначалия и } \\
\text { распределенных полномочий и ответственности. }\end{array}$ \\
\hline Функциональный потенциал. & Организационный потенциал. \\
\hline $\begin{array}{c}\text { Преобладание в основном социально } \\
\text { - экономических целей и критериев } \\
\text { функционирования. }\end{array}$ & $\begin{array}{c}\text { Цели - действенность, результативность в } \\
\text { ликвидации причин ЧС и их последствий; } \\
\text { критерии - минимизации времени достижения } \\
\text { целей, минимум потерь (жертв) при ликвидации } \\
\text { ЧС. }\end{array}$ \\
\hline
\end{tabular}

${ }^{1}$ Шульи В.Л., Кульба В.В., Шелков А.Б., Чернов И.В. Методы планирования и управления техногенной безопасностью на основе сценарного подхода // Национальная безопасность/Nota Bene. - 2013, №2 (25). 
Планирование и управление процессами предупреждения и ликвидации последствий техногенных катастроф должно охватывать весь круг проблем, касающихся чрезвычайных ситуаций, наиболее важными из которых являются этапы прогнозирования, противоаварийного упреждающего планирования и оперативного управления ликвидацией причин и последствий ЧС в условиях высокой степени неопределенности.

В настоящей работе основное внимание уделено анализу эффективности методов и механизмов решения задач планирования и оперативного управления комплексами мероприятий по предупреждению и ликвидации причин и последствий техногенных катастроф и чрезвычайных ситуаций различного типа.

\section{1. Анализ особенностей планирования процессов предупреждения и ликвидации причин и последствий техногенных катастроф и чрезвычайных ситуаций}

В отличие от традиционных систем планирования и управления ${ }^{2} 3$, которые призваны рассматривать стратегические задачи в течение достаточно длительного периода, системы управления в условиях ЧС должны действовать в реальном масштабе времени.

Принципиальными особенностями процессов планирования и управления ликвидацией последствий техногенных катастроф и чрезвычайных ситуаций являются:

1. частичная предсказуемость серьезных проблем и возможностей их решения;

\footnotetext{
${ }^{2}$ Архипова Н.И., Кульба В.В., Косяченко С.А., Чанхиева Ф.Ю., Шелков А.Б. Организационное управление. - М.: Изд - во РГГУ, 2007.

${ }^{3}$ Информационное обеспечение систем организационного управления (теоретические основы). В 3 - х частях. Часть 1. Методологические основы организационного управления. / Под ред. Е.А. Микрина и В.В. Кульббы. - М.: Изд - во физ. - мат. лит., 2011.
}

2. частичная предсказуемость очагов возникновения и сценариев развития ЧС;

3. слабая предсказуемость масштаба и времени возникновения ЧС;

4. непредсказуемость ряда неблагоприятных событий и ситуаций, обусловленных возникновением и характером развития ЧС (наличие стратегических неожиданностей).

Этими условиями диктуется необходимость преимущественного использования методов планирования и управления, основанных на предвидении проблем, ситуаций и событий, принятии гибких экстренных решений, ориентированных на внешнее окружение системы (природная среда, условия жизнеобеспечения населения и персонала предприятий и организаций, социально - политическая обстановка и т. д.). Такие методы могут быть эффективно реализованы в рамках единой современной концепции стратегического, тактического планирования и оперативного управления.

Как известно, под планированием понимается целенаправленный, организованный и непрерывный процесс выделения различных элементов и аспектов объекта управления, определения их состояния и взаимодействия в данное время, прогнозирования их развития на некоторый период в будущем, а также составления и программирования набора действий и ресурсов для достижения желаемых результатов.

Рассмотрим задачу превентивного планирования мероприятий по предупреждению и ликвидации причин и последствий техногенных катастроф и чрезвычайных ситуаций.

Процесс планирования должен предусматривать разработку комплекса мер (действий) по предотвращению возможной ЧС, а в случае ее наступления - мер по эффективной ликвидации последствий.

Целью превентивных мер предупреждения возможной ЧС на объекте является обеспече- 
ние желаемого его состояния, исключающего или смягчающего возникновение и развитие ЧС, заданной графом $G$, достижение которого требует разработки и реализации комплекса мероприятий, обеспечивающих безопасность объекта. Эти мероприятия необходимо осуществлять с привлечением необходимых ресурсов, сил и средств с учетом их наличия, вариантов размещения и затрат по вводу в действие. Мероприятия по предупреждению ЧС, направленные на достижение необходимого уровня безопасности региона ЧС, представим в виде орграфа $Q=(D, L)$, в котором множеству вершин $D$ соответствуют мероприятия (задачи), обеспечивающие достижение желаемой цели, а дугам $L$ графа - взаимосвязи между мероприятиями, определяющие последовательность их реализации. Дуги графа $Q$ ориентированы от мероприятий (задач), являющихся исходными для обеспечения безопасности объекта к желаемой конечной цели, которая представляется вершиной $D_{0}$. Граф $Q$ можно представить в виде матрицы $Q=\left\|q_{m n}\right\|, m, n=\overline{1, N}$ , где $q_{m n}=1$, если $n$ - е мероприятие предшествует $m$ - му мероприятию, и $q_{m n}=0$ в противном случае. Граф $Q$ тесно связан с исходным графом $G(S, B)$ и строится на основе анализа последнего. Для этого в графе $G$ выделяются конечные события, достижимые из вершины $S_{0}$, а также дуги, связывающие эти события. Каждой такой дуге ставятся в соответствие необходимые мероприятия $D=\left\{D_{m}, m=\overline{1, N}\right\}$, реализация которых приводит к достижению поставленной цели $D_{0}$. Построенный таким образом интегрированный граф $Q$ соответствует комплексу превентивных мер предупреждения ЧС, возможное развитие которой определяется заданным графом $G$. Взаимодействующие графы $G$ и $Q$ отражают существенные стороны общего процесса планирования превентивных мер по предупреждению возможной ЧС в регионе.

Таким образом, оптимальное планирова- ние превентивных мер по предупреждению ЧС в регионе заключается в выборе комплекса превентивных мероприятий, представленных в графе $Q$, для предотвращения возникновения и развития чрезвычайных событий, представленных в графе $G$.

Эффективный превентивный план формируется на основе оптимального распределения ресурсов, сил и средств различных видов, необходимых для реализации мероприятий с целью максимально возможного блокирования развития чрезвычайной ситуации в рассматриваемом регионе.

При планировании оперативных мероприятий в случае возникновения чрезвычайной ситуации и ее возможного развития в соответствии с полученным сценарием вместо графа $Q$ должен использоваться граф $W=(A, E)$ оперативных мероприятий по ликвидации последствий чрезвычайной ситуации. Граф $W$ также тесно связан с графом $G$ и определяет набор взаимосвязанных мероприятий чрезвычайного режима, обеспечивающих ликвидацию причин и последствий ЧС. Он также строится на основе результатов анализа возможностей блокирования дуг графа $G$, и его отличие от графа $Q$ состоит в содержании, составе и последовательности реализации оперативных мероприятий, свойственных чрезвычайному режиму.

Основными критериями формирования оптимальных превентивных планов по предупреждению и ликвидации последствий чрезвычайных ситуаций с использованием представленных графами $G$ и $Q(W)$ процессов являются: минимум людских потерь и ущербов, выраженных в стоимости материальных ценностей; минимум общих затрат на реализацию превентивных мероприятий по предупреждению ЧС; минимум общего времени реализации оперативных мероприятий по ликвидации последствий ЧС. В качестве ограничений используются ограничения на 
общие объемы ресурсов; сил и средств, выделенных для реализации мероприятий; на наличие необходимых сил и средств в пунктах их дислокации; структурные ограничения на связи чрезвычайных событий и проводимых мероприятий и др.

Общая задача формирования оптимального превентивного плана предупреждения чрезвычайной ситуации при заданных процессах $G$ и $Q$ формулируется следующим образом: необходимо выбрать множество блокируемых дуг графа $G$ путем назначения соответствующих превентивных мер из графа $Q$, обеспеченных необходимыми типами ресурсов и размещенных в заданных пунктах дислокации, обеспечивающих экстремум выбранного показателя эффективности.

Сформулируем основные критерии эффективности формирования оптимальных превентивных планов предупреждения чрезвычайных ситуаций. В зависимости от типов чрезвычайных ситуаций в регионе предлагается в качестве целевых использовать следующие функции.

Возможные людские потери определяются функцией вида:

$$
V_{0}=\sum_{i=0}^{I} \sum_{j=i+1}^{I} v_{i j} x_{i j}
$$

где $v_{i j}$ - людские потери от развития ЧС в соответствии с дугой $(i, j)$ и наступлением события $V_{j} ; X_{i j}=0$, если дуга $(i, j)$ блокируется и $X_{i j}=1-$ в противном случае.

Возможный ущерб определяется аналогичным образом, т. е.

$$
U_{0}=\sum_{i=0}^{I} \sum_{j=i+1}^{I} u_{i j} x_{i j}
$$

где $u_{i j}$ - ущерб, выраженный стоимостью материальных ценностей, разрушаемых при возможном развитии событий в соответствии с дугой $(i, j)$.

Общие затраты на ввод в действие ресурсов (сил и средств) при реализации превентивных мер, направленных на предупреждение ЧС в регионе, могут быть определены следующим образом:

$$
C_{0}=\sum_{i=0}^{I} \sum_{j=i+1}^{I} c_{i j} x_{i j}
$$

Здесь $C_{i j}$ - затраты на транспортировку и ввод в действие ресурсов (сил и средств) различных типов из пунктов размещения для проведения мероприятий по блокированию дуги $(i, j)$;

$$
c_{i j}=\sum_{m \in M_{i j}} \sum_{f=1}^{F} \sum_{k=1}^{K} d_{k f}^{(i, j)} P_{m k}^{(i, j)},
$$

где $P_{m k}^{(i, j)}$ - требуемое количество ресурсов $k$ го типа $(k=\overline{1, K})$ для реализации $m$ - го мероприятия (вершины графа $Q$ ) по блокированию дуги $(i, j)$ графа $\mathrm{G} ; d_{k f}^{(i, j)}$ - стоимость транспортировки и ввода в действие единицы ресурсов $k$ - го типа, размещенных в $f$ - м пункте, для блокирования дуги $(i, j)$ графа $G$.

Задача формирования оптимального превентивного плана предупреждения чрезвычайной ситуации на объекте, минимизирующего возможный суммарный ущерб в регионе формулируется следующим образом:

при ограничениях на:

$$
\min U_{0}\left(x_{i j}\right)
$$

- общую приведенную стоимость ресурсов (сил и средств), выделенных для реализации комплекса мероприятий по предупреждению ЧС в регионе на определенный период

$$
\sum_{i=0}^{I} \sum_{j=i+1}^{I} \sum_{m \in M_{i j}} \sum_{k=1}^{K} a_{k} P_{m k}^{(i, j)} x_{i j} \leq C
$$

где $C$-общая стоимость ресурсов, выделенных для данного региона в денежном выражении; $a_{k}$ - стоимость единицы ресурса $k$ - го типа; - количество ресурсов $k$ - го типа, выделенное для реализации мер по предупреждению ЧС:

\footnotetext{
${ }^{4}$ Сергиенко И.В. Математические модели и методы решения задач дискретной оптимизации. -Киев: Наук. думка, 1988..
} 


$$
\sum_{i=0}^{I} \sum_{j=i+1}^{I} \sum_{m \in M_{i j}} P_{m k}^{(i, j)} x_{i j} \leq R_{k},
$$

где $R_{k}$ - максимальное количество ресурсов (сил и средств), выделенных для региона;

- время проведения мероприятий по блокированию дуг графа $G$

$$
\max _{\left\{x_{i j}\right\}}\left\{T_{k p}^{(i j)} x_{i j}\right\} \leq T
$$

где $T_{k p}^{(i j)}$ - время выполнения работ критического пути по блокированию дуги $(i, j) ; T$ - заданный максимальный срок выполнения мероприятий.

Задача (4) - (7) является задачей целочисленного (булевого) программирования и может быть решена стандартными методами ${ }^{4}$. Возможны также постановки задач оптимизации планов проведения мероприятий по предупреждению ЧС в регионе с использованием целевых функций (1) и (3).

В настоящее время одним из наиболее перспективных направлений развития рассматриваемой методологии является типизация планирования и сценарного моделирования. Данное требующее отдельного рассмотрения направление предполагает выделение, анализ и паспортизацию типовых подклассов потенциально опасных объектов на основе утвержденной МЧС РФ классификации, включающей пять базовых классов, техногенные аварии на которых могут являться источниками возникновения федеральных (трансграничных), региональных, территориальных, местных и локальных чрезвычайных ситуаций соответственно.

Типизация (сведение всего многообразия планов, управляющих и контролирующих воздействий к достаточно ограниченному множеству) должна обеспечить возможность разработки и использования типовых механизмов и средств планирования и управления процессами предупреждения и ликвидации причин и последствий ЧС, а также значительную общность семантического и информационного содержания базовых (типовых) планов. Это, с одной стороны, обеспечит возможность использования при их составлении достаточно ограниченного множества информационных элементов, с другой - значительную инвариантность для органов управления различного уровня и ведомственной принадлежности, что должно упростить согласованную реализацию системы планов в различных режимах.

\section{2. Анализ особенностей оперативного управления в условиях чрезвычайных ситуаций}

Ход и результаты работ по ликвидации ЧС во многом зависят от эффективности работы системы оперативного управления. В свою очередь, эффективность процесса принятия, реализации и корректировки оперативных решений определяется умением руководства сосредоточить основные усилия на главных направлениях ликвидации причин ЧС и еe последствий.

Важной проблемой совершенствования системы оперативного управления является разработка методов принятия оптимальных решений и планов операций для ЧС в целом, а также и для отдельных стратегических ситуационных зон (СС3) или их совокупностей. Принимаемые в условиях ЧС управленческие решения - основа оперативного управления. В соответствии с ними строится вся последующая деятельность органов управления по ликвидации ЧС. Принятие оперативных решений в данных условиях является творческой и ответственной задачей. Суть ее состоит в том, чтобы в соответствии с поставленной целью, базовыми планами и складывающейся обстановкой определить общий замысел операции по ликвидации ЧС, конкретные меры ее реализации в каждой СС3, порядок взаи- 
модействия сил и средств при их выполнении и необходимое обеспечение. Оперативные решения принимает руководитель работ, и он же несет за них персональную ответственность. В подготовке данных для принятия оперативных решений участвуют почти все сотрудники органов управления в соответствии с их функциональными обязанностями (члены штаба и руководители СС3). В ходе реализации оперативных решений они могут заменять друг друга.

Оперативные управленческие решения в условиях ЧС должны удовлетворять требованиям своевременности, обоснованности и однозначности понимания исполнителями, а также категоричности их реализации.

Принимая управленческие решения в условиях ЧС, следует учитывать следующие основные противоречия:

- между сложностью оценки ситуации и принятия оперативных решений и практически нулевыми ресурсами времени для их реализации;

- эволюцией в направлении все большей специализации при решении задач в СС3 и необходимостью общей системной интеграции при принятии управленческих решений в штабе;

- необходимостью централизации управления, обеспечивающей взаимоувязку действий, сил и средств, а также оперативность координации и управления, и потребностями децентрализации, которая гарантирует устойчивость в управлении, повышает оперативность решения локальных задач и инициативу руководителей CC3;

- обоснованностью принимаемых решений и оперативностью управления.

Разрешение указанных противоречий является целью совершенствования системы оперативного управления в условиях ЧС.

Необходимо также учитывать существен- ную разницу между характеристиками задач планирования работ по предупреждению и ликвидации последствий ЧС и задач оперативного управления, решаемых в чрезвычайных условиях.

Задачи планирования, как правило, характеризуются большим объемом работ и большой размерностью, причем объем работ меняется во времени незначительно. Частота их решения обычно невелика, периоды решения регламентированы, а время решения не является лимитирующим фактором. Частота изменения исходных данных относительно невелика, а их достоверность мала. Поэтому точность решения задач планирования определяется в основном достоверностью исходных данных.

Задачи оперативного управления в условиях ЧС характеризуются случайными моментами времени поступления заявок на их решение. В среднем объем вычислительной работы для решения задач оперативного управления небольшой, частота же поступлений задач в обслуживающие узлы велика. Время решения, хотя, как правило, и невелико, но является лимитирующим фактором. Частота изменений исходных данных наоборот велика, а их достоверность мала. Точность решения задач оперативного управления обычно определяется не только достоверностью данных, но и качеством алгоритмов решения задач данного класса.

Следует отметить, что в условиях ЧС возникает по сути дела новый класс задач стратегического управления, которые по своим характеристикам являются задачами планирования (значимость, размерность, объем и т.д.), но должны решаться в условиях ограниченного времени (как задачи оперативного управления).

Весьма важной и специфической особенностью является проблема оценки эффективности функционирования систем оперативного управления ЧС. В отличие от систем управле- 
ния промышленностью, сельским хозяйством, торговлей и бытовым обслуживанием и т.д., эффективность которых оценивается производительностью, качеством выпускаемой продукции, прибыльностью, затратами и прочими факторами, эффективность функционирования систем оперативного управления в условиях ЧС должна оцениваться действенностью и результативностью.

Действенность является стратегическим показателем и служит для оценки степени достижения системой поставленных перед ней целей, уровня завершенности требуемых работ. При оценке действенности необходимо учитывать, выполняются ли все требуемые работы в соответствии с характером развития ЧС и выработанной стратегией ликвидации ее последствий, эффективны ли они, достигаются ли требуемые результаты и вовремя ли они реализуются. Чтобы измерить действенность, необходимо сравнить степень выполнения плановых работ, т.е. сравнить то, что требовалось сделать с фактически достигнутым.

Результативность является оперативно - тактическим показателем и представляет собой, по сути дела, упрощенный показатель действенности. Показатель результативности отражает текущие характеристики уровней выполнения работ в системе в целом и по отдельным СС3.

Одним из основных требований к системе управления в условиях ЧС является требование ее высокой оперативности. Под оперативностью (быстродействием) системы управления принято понимать ее способность обеспечивать своевременное решение задач управления в соответствии с целями системы и имеющимися ограничениями. Оперативность оценивается временем между возникновением ситуации, требующей вмешательства соответствующих органов управления, и временем ее ликвидации. Требование к быстродействию систем оперативного управления ликвидацией
ЧС существенно отличается от аналогичного требования к системам управления в промышленности или экономике. Дело в том, что при управлении ликвидацией ЧС недостаточно ориентироваться лишь на календарное время реализации различных мероприятий - следует учитывать их относительное (по отношению к темпу развития ЧС) время.

Большую роль в процессах оперативного управления ликвидацией ЧС играет тщательный учет баланса времени, заключающийся в сопоставлении имеющихся временных ресурсов с динамикой развития ЧС и вычислении его резерва или дефицита. С этой целью при управлении ликвидацией ЧС следует рассматривать два вида времени - располагаемое и требуемое, которые находятся в противоречии. Располагаемое время - время, в течении которого необходимо осуществить какое - либо мероприятие, чтобы обеспечить его успех. Это то время, которое определяется динамикой развития ЧС и ее негативных последствий или устанавливается вышестоящим руководством в виде некоторого директивного показателя, обычно также зависящего от динамики развития ЧС. Требуемое время - время, необходимое для проведения некоторого мероприятия с учетом уровня подготовки, обеспеченности и состояния системы управления.

Благоприятным считается такой временной баланс, когда требуемое время не превышает располагаемого. Чем больше разница между располагаемым и требуемым временем, тем больше резерв времени для компенсации непредвиденных обстоятельств, элементов случайности и неопределенности. В том случае, когда требуемое время оказывается больше располагаемого, образуется дефицит времени, который не позволяет выполнить запланированный объем работ или выполнить с той эффективностью, которая предполагалась. Времена и редко бывают жестко детерминированы. Обычно они рассматриваются как 


\section{Тренды и управление - № 2 (2)•2013}

вероятностные величины. Как правило, их следует считать математическими ожиданиями (средними значениями) соответствующих времен, учитывая, что фактическое время в силу разнообразных обстоятельств может колебаться относительно этих средних значений. Помимо обычных типов резервов (таких, как рассмотренные выше временные и ресурсные) при решении задач оперативного управления в условиях ЧС следует использовать и другие типы резервов, а именно структурно - технологический, природно - климатический и эколого - физиологический.

Наличие структурно - технологического резерва позволяет эффективно управлять имеющимися силами и средствами при потере работоспособности рядом элементов системы. Под структурно - технологическим резервом некоторого элемента системы а понимается множество элементов R(a), объединенных структурно - технологическими связями с теми же элементами, что и элемент а, и способных выполнять технологические операции (либо некоторые из них) из множества О(a). Здесь $\mathrm{O}(\mathrm{a})$ - множество технологических операций по ликвидации последствий ЧС, которые могут быть выполнены элементом а.

Природно - климатический резерв подразделяется на два основных типа. Природно - климатический резерв первого типа дает возможность существования организации в условиях невозможности выполнения ею основных функций за счет реализации некоторого множества других функций (детерминированного или недетерминированного). Обычно эффективность от их реализации для данной организации ниже, чем от реализации основных функций. Природно - климатический резерв второго типа позволяет организации снижать затраты на реализацию некоторого множества функций в течение определенного периода времени благодаря обеспечению в этот период достаточно удовлетворительных условий их реализации в конкретных природно - климатических условиях.

Примером использования природно - климатического резерва первого типа является довольно обычная для сельскохозяйственных предприятий ситуация, когда из - за заморозков погибают посевы ряда теплолюбивых культур. При наличии ресурсов различного типа предприятие повторно обрабатывает почву и производит посадку на этих площадях таких культур, которые хотя и не являются профилирующими для данного хозяйства, однако могут быть выращены в сложившихся условиях. Необходимым условием существования резерва такого типа является превышение дохода от реализации новых культур над затратами на их производство.

Примером природно - климатического резерва второго типа служит возможность использования туристических палаток вместо стационарных помещений в качестве временного жилья при проведении эвакуационных работ в условиях соответствующего климата и т. д.

Эколого - физиологический резерв также бывает двух типов. Первый тип данного резерва - это способность живого организма (в частности человека) противостоять изменениям условий среды обитания без последующего ухудшения состояния своего здоровья. Под эколого - физиологическим резервом второго типа понимается возможность стабилизации ситуации и приведения ее к нормальным условиям без вмешательства человека за счет использования только природных факторов. Величины резервов первого типа могут быть установлены с помощью медицинских исследований возможностей организма человека и определения нормативов его пребывания во вредных условиях (радиация, шум, вибрация и т. д.). Резервы второго типа определяются при изучении возможностей самоочистки рек, атмосферы и т. д. 
Управление безопасностью

С целью повышения эффективности принимаемых решений и контроля за их реализацией функции системы оперативного управления ликвидацией последствий крупных ЧС целесообразно распределять по следующим составляющим основу базового оперативного плана группам конкретных мероприятий.

1. Оповещение и информирование населения о возникновении чрезвычайной ситуации, включающее оперативное оповещение населения, администрации региона и членов комиссии по ЧС о факте (угрозе) ее возникновения и адресах пунктов сбора для организации работ по ликвидации ее последствий, а также информирование населения о масштабах и последствиях ЧС с возможной организацией для этой цели специализированного пресс - центра.

2. Организация и ведение общей и специальной разведки зон разрушений с целью получения точных данных о сложившейся обстановке в результате возникновения ЧС. Первый вид разведки проводится для определения общего характера и ориентировочного объема разрушений и повреждений в очаге поражения, установления уровней радиации, площадей распространения пожаров, выявления скоплений ядовитых и отравляющих веществ, уточнения маршрутов движения транспорта и эвакуации пораженных, выяснения условий проведения спасательных работ и т.д. Специальная разведка имеет целью получить более полные и точные сведения о завалах и разрушенных строениях, потенциальных и реальных человеческих потерях, характере разрушений зданий и сооружений, состоянии сетей коммунального и энергетического хозяйства, а также наиболее безопасных путях подхода к объектам работ.

3. Мобилизащия сил и средств для ликвидаџии последствий ЧС. Мобилизация сил быстрого реагирования в составе первого эшелона проводится в целях сбора, организации и доставки в зоны поражения реальных сил и средств региона (города) в самые кратчайшие сроки с момента возникновения ЧС для проведения спасательных и аварийно - восстановительных работ. Состав, виды и распределение сил и средств быстрого реагирования определяются в превентивных планах ликвидации последствий ЧС, являющихся основой для формирования и ввода в действие оперативных планов спасательных и других неотложных работ в зонах поражения.

4. Охрана важных государственных объектов и очепление разрушенных участков и районов. Выполнение данной функции призвано обеспечить охрану важных государственных объектов сосредоточения материальных ценностей (банки, ювелирные и другие магазины, финансовые учреждения, склады, оптовые базы и т. д.) и личного имущества граждан, предотвратить мародерство и другие преступления. Зона бедствия оцепляется, как правило, личным составом полиции и внутренних войск, привлекаемыми для этой цели подразделениями армии и т. п.

5. Восстановление и организация систем и средств связи. Для решения данной задачи из мест постоянной дислокации в назначенные участки и районы выдвигаются подвижные узлы связи (при необходимости в зонах разрушения развертываются полевые средства связи). Восстановление поврежденных средств и систем связи осуществляется сформированными бригадами специалистов.

6. Выделение зон и участков ведения спасательных и других неотложных работ. Эффективность организации разведки и мобилизации сил и средств тесно связана с выделением стратегических ситуационных зон (СС3) для ведения разведывательных, 
спасательных и других неотложных работ. От разделения региона (города) на зоны поражения зависят определение потребности в силах и средствах для ведения аварийно - спасательных работ, выбор рациональных маршрутов движения транспортных средств, определение пунктов сосредоточения спасательных формирований. В общем случае ущерб от крупных ЧС может быть неравномерным по районам города, а внутри районов - по СС3.

7. Действия в чрезвычайном режиме направлены прежде всего на спасение пострадавших, оказавшихся в очаге ЧС. Конкретные меры и время их реализации во многом зависят от сложившихся на момент возникновения ЧС условий.

8. Организация комплексного ресурсного обеспечения групп и формирований, ведущъих спасательные, восстановительные и другие неотложные работы. В рамках выполнения данной функции предусматривается оперативная доставка формированиям и группам, ведущим спасательные и иные неотложные работы в зонах ЧС, дополнительного специального оборудования и инструментов, приборов и средств для эффективного ведения подобных работ. План распределения материальных ресурсов должен учитывать специфические особенности зоны поражения и степень разрушения объектов, а также нормативные требования к объему ресурсов, необходимому для эффективного проведения работ.

9. Эвакуация населения. Необходимость в эвакуации населения возникает в случае разрушения (угрозы разрушения) жилых зданий и массивов, выбросов сильнодействующих ядовитых веществ, охватывающих большие территории, при угрозе наводнений и селей, в результате разрушительного землетрясения.
10. Организациия медициинской помощии и стациионарной базы для госпитализации пострадавших является одной из наиболее приоритетных функций, поэтому и выделяется в отдельную систему оказания оперативной медицинской помощи населению, пострадавшему в результате возникновения ЧС. С этой целью и на основании данных о масштабах и последствиях ЧС привлекаются бригады скорой медицинской помощи, развертываются мобильные госпитали Центра медицины катастроф и создаются стационарные базы для госпитализации пострадавших на базе ближайших к очагу ЧС медицинских учреждений.

11. Организация обеспечения медикаментами и оборудованием спасательных и других неотложных работ и развернутых стацуионаров и клиник, организация эпидемиологического и ветеринарного контроля, обслуживание безвозвратных потерь и т.П. также относятся к основным функциям системы оперативного управления ликвидацией последствий крупных ЧС.

Превентивное планирование оперативных ответных действий, связанных с использованием сил и средств ликвидации последствий ЧС в регионе в чрезвычайном режиме имеет свои особенности, связанные с характером развития ЧС. Во многих случаях развитие ЧС происходит в течение достаточно короткого интервала времени (землетрясения, наводнения, взрывы, пожары), недостаточного для эффективного предотвращения (блокировки) наступления чрезвычайных событий, представленных в рассмотренном выше графе $G$.

В чрезвычайном режиме, как правило, необходимо в минимально короткий срок провести комплекс последовательно реализуемых аварийно - спасательных и других неотложных работ (АСДНР) в наиболее пострадавших объектах региона, связанных с ликвидацией последствий наступивших на них чрезвычайных 
событий $S^{j}$ графа G. Для постановки задачи оптимизации общего времени проведения АСДНР введем необходимые переменные и обозначения: $t_{m \mathrm{k}}^{j}-$ общее время использования $k$ - го типа ресурсов $(k=\overline{1, K})$ для проведения $m$ - го мероприятия $(m=\overline{1, M})$ комплекса АСДНР при ликвидации последствий $S_{j} \in S^{*}$ $; t_{m}^{j}=\max _{k}\left\{t_{m k}^{j}\right\}-$ общее время реализации $m$ - го мероприятия;

$x_{j}=\left\{\begin{array}{l}1-\text { если последствия событий } S j \text { ликвидируются } \\ \text { путем проведения комплекса АСДНР, } \\ 0-\text { в противном случае. }\end{array}\right.$

Задача минимизации максимального времени проведения АСДНР на объектах региона при ограничениях на величину конечных потерь, ущербов и затрат на ввод в действие необходимых сил и средств формулируется следующим образом:

$$
\min _{\left\{x_{j}\right\}} \max _{j} \sum_{m \in M_{j}} t_{m}^{j} x_{j}
$$

при ограничениях:

- на величину конечных людских потерь

$$
\sum_{j=1}^{J} V_{j}\left(1-x_{j}\right) \leq V_{\text {доп }}
$$

- на величину конечных ущербов

$$
\sum_{j=1}^{J} U_{j}\left(1-x_{j}\right) \leq U_{\text {доп }} ;
$$

- на общую стоимость выделенных ресурсов

$$
\sum_{j=1}^{J} \sum_{m \in M_{j}} P_{m k}^{j} x_{j} \leq R_{\text {доп }} ;
$$

- на гарантированное включение в план отдельных чрезвычайных событий ( $x_{j}=1, S_{j} \in S^{*}$, где $S^{*} \in S-$ подмножество событий, гарантировано включаемых в превентивный план проведения АСДНР);

- на общее число включаемых в план чрезвычайных событий

$$
\sum_{j=1}^{J} x_{j} \leq S_{\text {дир }} .
$$

Поставленная задача относится к числу задач целочисленного программирования и решается с использованием стандартных методов. Результаты решения поставленной задачи позволяют региональным органам управления получить опорный превентивный план действий в условиях наступления и развития ЧС в соответствии со сформированным региональным сценарием и поставленным ему в соответствие графом причинно - следственных связей чрезвычайных событий.

Рассмотрим постановку и методы решения некоторых задач оперативного управления силами и средствами в чрезвычайном режиме. Анализ отечественного и зарубежного опыта, связанного с планированием и проведением комплексов мероприятий по защите населения от последствий крупных ЧС различного типа, показывает, что общая их эффективность существенно зависит от качества формирования и реализации оперативных решений по развертыванию и вводу в очаги поражения необходимого количества аварийно - спасательных и восстановительных бригад (АСВБ), оказанию первой помощи и эвакуации пораженных, проведению своевременной широкомасштабной эвакуации населения из зоны (зон) экстренных мер защиты.

Необходимо отметить, что предлагаемая технология реализации аварийно - спасательных работ предусматривает непосредственный ввод аварийно - спасательных бригад постоянной готовности (АСБПГ) в пункты действий, а бригад другого типа - через промежуточные пункты развертывания (сосредоточения). Это обстоятельство обусловливает специфику 


\section{Тренды и управление - № 2 (2)•2013}

формирования оптимальных оперативных решений по переброске бригад указанного типа, связанную с определением исходной структуры сети перебросок, критериев оптимальности и соответствующих ограничений.

Потребность в специализированных бригадах различного типа определяется штабом управления силами и средствами (СиС) на основе данных разведки применительно к каждому пункту производства экстренных работ (действий) в выделенной стратегической зоне (CC3). Кроме того, известна дислокация СиС в зоне, подверженной действию ЧС, а также в соседних областях. Задана также ориентированная сеть с промежуточными узлами, позволяющая перебросить необходимые СиС (бригады) из пунктов дислокации (источников) в пункты действий (стоки). Требуется сформировать общий оперативный план (планы) переброски бригад в соответствии с заданным критерием эффективности, пропускной способности дуг сети и ограничениями на объем предложения источников и объем спроса в стоках.

Задачи формирования оперативных решений такого типа могут быть формализованы следующим образом.

Сеть перевозок может быть представлена в виде ориентированного графа $G=(N, A)$, где $N=N_{\alpha} \cup N_{\beta} \cup N_{\gamma}$ - множества источников, стоков и промежуточных узлов соответственно. Каждая дуга сети характеризуется верхней границей $U_{i j}$ потока $\Psi_{i j}$ через нее и стоимостью $C_{i j}^{s}$ единицы потока $s$ - го типа. Специфической особенностью сети перевозок применительно к переброскам аварийно - спасательных бригад является наличие бригад различного типа в $i$ - м исходном пункте дислокации. Обозначим предложение $i$ - го узла сети через $a_{i}^{s}, s=\overline{1, S}$ , а потребность $j$ - го пункта через $b^{s}$. В этих условиях задачи оптимизации оперативных перевозок могут быть сформулированы как задачи выбора структуры многопродуктового потока в сети $G$, связанного с определением оптимального множества потоков в ее дугах, т.е. множества $\left\{X_{i j}^{s}\right\}, s=\overline{1, S},(i, j) \in A$, где $X_{i j}^{s}$ - поток $s$ - го продукта по дуге $(i, j)$, интерпретируемый как число бригад s - го типа, перевозимых по дуге $(i, j)$.

Для обеспечения безопасности и высокой скорости переброски $s$ - й бригады по дуге $(i, j)$ должны быть выделены соответствующие силы и средства государственной инспекции по безопасности дорожного движения и военной автоинспекции, затраты на которые определим как $C_{i j}^{s}$. Тогда многопродуктовая или многоцелевая задача о перевозках бригад различного типа формулируется как следующая задача целочисленного линейного программирования:

$$
\min _{\left\{X_{i j\}}^{s}\right\}} \sum_{s=1}^{S} \sum_{(i, j) \in A} C_{i j}^{s} X_{i j}^{s},
$$

при условии, что

$$
\sum_{j} X_{i j}^{s}-\sum_{j} X_{j i}^{s}=a_{i}^{s},
$$

если узел $i$ является источником продукта $s$;

$$
\sum_{j} X_{i j}^{s}-\sum_{j} X_{j i}^{s}=0,
$$

если узел связи $і$ является промежуточным узлом;

$$
\sum_{i} X_{i j}^{s}-\sum_{i} X_{j i}^{s}=-b_{j}^{s}
$$

если узел ј является стоком продукта $s$;

$$
\begin{gathered}
\sum_{s} X_{i j}^{s} \leq U_{i j}^{s} ; \text { для всех }(i, j) \in A \\
X_{i j}^{s} \geq 0 \text { для всех } s \text { и }(i, j) \in A .
\end{gathered}
$$

В том случае, когдазначения $C_{i j}^{s}$ трудноопределяемы, можно положить $C_{i j}^{s}=1 \forall(i, j) \in A$. Тогда критерий (11) имеет смысл минимума суммы всех потоков перевозок, циркулирующих на дугах сети.

Применительно к ЧС типа общей радиа- 
ционной аварии на атомной электростанции коэффициенты $C_{i j}^{s}$ целесообразно интерпретировать как прогноз или использовать при оперативном управлении эвакуацией населения и сельскохозяйственных животных из зоны поражения. В этом случае необходимо минимизировать суммарную дозу облучения на выбранных маршрутах из мест эвакуации (убежища, учреждения, жилые дома, сборные пункты) до контрольно - пропускных пунктов (КПП) на границе зоны возможного опасного радиоактивного загрязнения. Выбор эффективной архитектоники, т. е. пространственного расположения (структуры) КПП может осуществляться с помощью оценки оптимальных значений критерия (11) для фиксированных вариантов структуры.

Необходимо отметить, что выбор пространственной структуры пунктов развертывания (сосредоточения) сил и средств различного типа и маршрутов их последующего ввода в загрязненную зону может быть также осуществлен путем последовательного решения задачи (11) - (16) для ряда выбранных рациональных вариантов.

Для большинства ЧС техногенного и природного характера наиболее адекватными для оптимального оперативного управления переброской сил и средств являются критерии временного типа. Достаточно эффективным критерием такого типа является минимум суммарного времени перевозок по дугам сети $G$. При этом общее время транспортировки $t_{i j}$ по дуге $(i, j)$ является некоторой функцией потока по данной дуге, т. е.

$$
t_{i j}=f_{i j}\left(\sum_{s} X_{i j}^{s}\right) .
$$

Критерий оптимизации перевозок в условиях (12) - (16) в этом случае будет иметь следующий вид:

$$
\min _{\left\{X_{i j}^{s}\right\}} \sum_{(i, j) \in A} f_{i j}\left(\sum_{s} X_{i j}^{s}\right) \text {. }
$$

Как правило, целевая функция (17), являет- ся выпуклой, однако ее определение и последующее решение задач оптимизации являются существенно более сложными по сравнению с задачами типа (11) - (16). Поэтому представляется целесообразным рассмотрение достаточно простых в вычислительном отношении постановок задач оптимального оперативного управления перевозками по критериям временного типа, решение которых может быть получено в реальном масштабе времени в информационно - управляющих системах быстрого развертывания в соответствии с обстановкой, складывающейся при возникновении и развитии ЧС. В таких системах существенным образом могут быть использованы экспертные данные о пессимистических оценках общего времени переброски специализированных бригад из исходных пунктов в конечные имеющимися транспортными средствами.

Рассмотрим задачу формирования оптимального по конечному времени реализации оперативного плана доставки необходимого количества аварийно - спасательных бригад в пункты производства работ при ограничениях на число бригад и транспортных единиц различного типа в местах дислокации и транспортную доступность пунктов доставки из пунктов дислокации.

Пусть:

- $j=\overline{1, J}$ - пункты доставки бригад (производства работ), заданные своими координатами;

$-s=\overline{1, S}$ - типы аварийно - спасательных подразделений (пожарные команды, механизированные команды, медицинские бригады, бригады химзащиты и т. д.);

- $V_{j}^{s}, j=\overline{1, J} ; s=\overline{1, S}-$ потребность в числе спасательных бригад $s$ - го типа в $j$ - м пункте;

- $i=\overline{1, I}$ - пункты исходной дислокации бригад; заданные своими координатами.

$-V_{i}^{s}-$ наличие бригад $s$ - го типа в $i$ - м пункте, 


\section{Тренды и управление - № 2 (2)•2013}

- $\left\|d_{i j}^{n}\right\|$ - матрица смежности между пунктами дислокации и доставки для $n$ - го транспортного средства $(n=\overline{1, N})$;

$-V^{n_{s}}$ - количество транспортных средств $n$ - го типа для переброски одной бригады $s$ го типа;

$-t_{i j}^{n_{s}}-$ пессимистическая оценка времени переброски из $i$ в $j$ бригады $s$ - го типа, выполняемой транспортным средством

$n$ - го типа.

Введем следующие переменные:

$x_{j}=\left\{\begin{array}{l}1-\text { если реализуется переброска } i \text {-й в } j \text {-ю бригады } \\ S \text {-го типа } n \text {-м транспортным средством, } \\ 0-\text { в противном случае. }\end{array}\right.$

$V_{i j}^{n_{s}}$ - число бригад $s$ - го типа перебрасываемых из $i$ в $j$ транспортным средством $n$ - го типа.

Рассмотренная выше задача формулируется следующим образом:

$$
\min _{\left\{X_{i j}^{n_{s}}\right\}}\left[\max _{j}\left(\max _{\{(i, s, n)\}}\left\{t_{i j}^{n_{s}} X_{i j}^{n_{s}}\right\}\right)\right]
$$

при ,огранцујениях:

- на объем потребностей в пунктах доставки;

$$
\sum_{n=1}^{N} \sum_{i=1}^{I} V_{i j}^{n_{s}} X_{i j}^{n_{s}} \geq V_{j}^{s}, \quad j=\overline{1, J}, \quad s=\overline{1, S}
$$

- на наличие транспортных средств в пунктах дислокации

$$
\sum_{j} V_{i j}^{n_{s} I n_{2 j}^{n_{s}}} X_{i j}^{n_{s}} \leq C_{i}^{n_{s}}, i=\overline{1, I}, n=\overline{1, N}, S=\overline{1, S}
$$

- на наличие сил и средств в местах дислокации

$$
\sum_{n=1}^{N} \sum_{j=1}^{J} V_{i j}^{n_{s}} X_{i j}^{n_{s}} \leq V_{i}^{s}, \quad i=\overline{1, I}, \quad s=\overline{1, S}
$$

- на транспортную доступность пунктов $j$ из пунктов $i$ в соответствии с матрицей $\left\|d_{i j}^{n}\right\|$ :

$$
X_{i j}^{n_{s}}=0
$$

для заданных пар $(i, j), n=\overline{1, N}$;

- на целочисленность переменных

$$
V_{i j}^{n_{s}} \in\{0,1,2, \ldots\} .
$$

Задача (18) - (23) относится к классу задач комбинаторного типа и может быть эффективно решена методами прямого либо направленного перебора с использованием современных персональных ЭВМ.

По результатам действий спасательных бригад в зоне ЧС для каждого из пунктов $j$ $(j=\overline{I, J})$ определяется число пострадавших, которым не может быть оказана медицинская помощь в районе бедствия, и которые должны быть госпитализированы в лечебные учреждения, включенные в предварительный список превентивной готовности и располагающие соответствующими ресурсами и средствами лечения. Как правило, для эвакуации такого контингента применяют высокоскоростные транспортные средства, которые, в случае необходимости, используются в сочетании с другими видами транспорта.

В этих условиях целесообразно сформировать план оперативной эвакуации пострадавших из пунктов $j$ в пункты $(j=\overline{I, J})$ через множество возможных перевалочных пунктов $p=\overline{1, P}$ с учетом возможностей транспортных средств и пунктов приема пострадавших. Такой план должен минимизировать максимальное время доставки пострадавших в пункты госпитализации $i=\overline{1, J}$.

Задача формулируется следующим образом:

$$
\min _{\left\{X_{j p i}^{n n^{n}}, X_{j i}^{n}\right\}}\left\{\max _{j}\left[\min \left(t_{j p i} X_{j p i}^{n n^{\prime}}, t_{j i} X_{j i}^{n}\right)\right]\right\} .
$$

при ограничениях:

- на количество койко - мест в пунктах 
приема пострадавших (включая койко - места постоянной готовности ближайших к очагу ЧС лечебно - профилактических учреждений)

$$
\sum_{n=1}^{N} \sum_{j=1}^{J} V_{j i}^{n} X_{j i}^{n}+\sum_{\left(m \prime^{\prime}\right)} \sum_{j=1}^{J} \sum_{p=1}^{P} V_{j p i}^{n n^{\prime}} X_{j p i}^{n^{\prime}} \leq V_{i}, \quad i=\overline{1, I} ;
$$

- на потребность в вывозе пострадавших в пунктах $j$

$$
\sum_{n=1}^{N} \sum_{i=1}^{I} V_{j i}^{n} X_{j i}^{n}+\sum_{\left(n n^{\prime}\right)} \sum_{i=1}^{I} \sum_{p=1}^{P} V_{j p i}^{n n^{\prime}} X_{j p i}^{n n^{\prime}}=V_{j} ;
$$

- на наличие транспортных средств в пунктах $j$

$$
\sum_{i=1}^{I} V_{j i}^{n} n_{j i}^{n} X_{j i}^{n}+\sum_{p=1}^{P} V_{j p i}^{n n '} v_{j p}^{n} X_{j p i}^{n n^{\prime}} \leq C_{j}^{n}, \quad n=\overline{1, N}, j=\overline{1, J} ;
$$

- на наличие транспортных средств в пунктах

$$
\begin{aligned}
& \sum_{i=1}^{I} V_{j p i}^{n n^{\prime}} n_{p i}^{n^{\prime}} X_{j p i}^{n n^{\prime}} \leq C_{p}^{n^{\prime}}, n^{\prime}=\overline{1, N}, p=\overline{1, P}, \\
& \text { - на целочисленность переменных }
\end{aligned}
$$

$$
X_{j i}^{n}, X_{j p i}^{n n^{\prime}} \in\{0,1,2, \ldots\}
$$

где $X_{j i}^{n}=1$, если реализуется эвакуация из $j$ в $i$ непосредственно $n$ - м транспортным средством, и $X_{j i}^{n}=0$, если нет; $X_{j p i}^{n n '}=1$, если реализуется эвакуация из пункта $j$ в пункт $i$ через пункт $p$ последовательно транспортными средствами $n$ и $n^{\prime}, X_{j p i}^{n n^{\prime}}=0$, если нет; $V_{j i(j p i)}^{n\left(n n^{\prime}\right)}$ - число эвакуируемых единиц из пункта $j$ в пункт $i$.

Задача (24) - (29) относится к тому же классу комбинаторных задач, что и предыдущая. Ее постановка и решение целесообразны также для случаев эвакуации населения из опасной зоны (зон) в случае угрозы его безопасности в места, определенные в превентивном плане ликвидации последствий ЧС.

Расширение моделирующих возможностей задачи (24) - (29) и, соответственно, повышение эффективности ее использования при управлении оперативными перевозками пострадавших достигаются путем ввода в модель результатов группового разделения пострадавших в процессе медицинской сортировки (пораженные с несовместимыми для жизни, крайне тяжелыми повреждениями; пораженные с тяжелыми повреждениями, сопровождающимися нарастающими расстройствами жизненно - важных функций организма; пораженные с повреждениями средней степени тяжести; легкопораженные с благоприятным прогнозом для жизни и трудоспособности); данных о возможностях и расположении базовых, резервных и вспомогательных стационарных медицинских учреждений, типов специализированных транспортных средств и т.п.

\section{3. Особенности использования сценарного анализа в процессах управления предупреждением и ликвидацией последствий ЧС}

Планирование находится в тесной взаимосвязи с прогнозированием и реализацией планов, которые должны рассматриваться не раздельно, а как взаимосвязанные части единого процесса управления риском.

Полный цикл планирования и управления риском возникновения техногенных катастроф и чрезвычайных ситуаций, а также ликвидацией их последствий включает:

- прогноз риска возникновения и тяжести последствий ЧС путем формирования сценариев развития ситуации;

- формирование целей и критериев управления риском;

- стратегическое (долгосрочное) планирование превентивных мероприятий;

- тактическое (текущее) планирование альтернативных ответных действий на возникающие угрозы ЧС;

- стратегическое и оперативное управление в условиях ЧС. 


\section{Тренды и управление - № 2 (2)•2013}

Риск возникновения и развития ЧС природного и техногенного типов прогнозируется на основе построения сценариев возникновения и развития таких ситуаций ${ }^{5}$. Сформированный сценарий позволяет адекватно отразить процесс развития ЧС, разработать стратегии организации и реализации превентивных и оперативных мер противодействия ЧС, сформировать стратегические и тактические планы действий, провести качественный анализ последствий, а также прогнозировать предполагаемый ущерб и потери.

Совокупность локальных сценариев для объектов, расположенных на определенной территории (районе, городе, области) является основой для построения не только региональных сценариев развития ЧС, но и соответствующих графов причинно - следственных связей чрезвычайных событий (ГПСС) для выделенного региона. Вершинами ГПСС регионального уровня являются чрезвычайные события на объектах региона, а дугами - причинно следственные связи между ними. Могут быть выделены два типа таких графов. Граф, построенный для некоторой общерегиональной ЧС, последствия которой распространяются на различные объекты и административно территориальные единицы, удаленные друг от друга в пределах региона на достаточно большое расстояние, определим как граф с одним общим источником $S_{0}$ чрезвычайной ситуации. Граф, построенный для множества объектов, каждый из которых потенциально является источником возникновения и развития ЧС определенного типа, определим как граф со многими источниками $\left\{s_{o}^{k}, k=\overline{1, K}\right\}$ чрезвычайных ситуаций.

Таким образом, в общем случае имеется ГПСС с одним или многими источниками

\footnotetext{
${ }^{5}$ Модели и методы анализа и синтеза сценариев развития социально - экономических систем: в 2-х кн. / под ред. В.Л. Шульц̧а, В.В. Кульбыл. - М.: Наука 2012 .
}

ЧС, элементы множества $S$ вершин которого (события) привязаны к конкретным населенным пунктам рассматриваемого региона, а элементы множества дуг $B$ отражают взаимное влияние возможных чрезвычайных событий в регионе. В общем случае такой граф $G$ является сетью.

Так как дугам регионального графа соответствуют процессы развития чрезвычайных событий в регионе, то блокирование некоторой дуги приводит к ограничению или полной ликвидации развития последующих событий в графе $G$.

Блокирование дуг осуществляется назначением на них необходимых и достаточных ресурсов различного типа, обеспечивающих предупреждение или ограничение процессов развития ЧС.

В результате блокирования дуг графа $G$, исходя из рационального распределения имеющихся в регионе ресурсов, сил и средств, будет получен подграф $G^{*}=\left(S^{*}, B^{*}\right)$ графа $G$, множество вершин которого $S^{*}$ представляет собой множество событий в регионе, а множество дуг $B^{*}$ причинно - следственные связи между событиями, развитие которых не удалось предотвратить и наступление которых связано с определенными ущербами и потерями.

Основной особенностью использования сценарного подхода в процессах планирования и управления предупреждением и ликвидацией последствий техногенных ЧС является необходимость расширения его возможностей для проведения комплексного многостороннего опережающего анализа складывающейся ситуации.

Одной из важнейших особенностей техногенных ЧС как предметной области является достаточно мощная нормативно - регламентная база, разработка и совершенствование которой непрерывно ведется по целому ряду направлений, таких как промышленная, транспортная, пожарная, радиационная, химическая, 
энергетическая, экологическая, социальная, общественная безопасность, безопасность жизнедеятельности, паспортизация и сертификация потенциально опасных объектов, медицина катастроф и т.п. Это позволяет существенно повысить эффективность методологии сценарного анализа в управлении ликвидацией последствий ЧС и изменить уже ставшую традиционной идеологию и технологию формирования и исследования имитационных моделей.

Широко применяемый в настоящее время для исследования проблем повышения эффективности развития социально - экономических систем так называемый функциональный подход, когда, например, за основу берется одна или несколько «близких» по своей природе конкретных функций управления, оказывается недостаточно эффективным при решении задач управления ликвидацией последствий техногенных ЧС. Основной причиной такого положения является необходимость более широкого, комплексного анализа развития ситуации и учета синергического характера развития неблагоприятных процессов и явлений, что существенно расширяет границы исследуемой предметной области и за счет этого снижает эффективность традиционного подхода.

Использование функционального подхода в данных условиях порождает объективные сложности объединения знаний экспертов в различных предметных областях в единую картину, позволяющую проводить комплексный многосторонний анализ состояния и тенденций развития сложившейся ситуации. Это обусловлено прежде всего различием профессиональных языков (терминологий) экспертов, противоречивостью процедур и несовпадением результатов экспертных оценок, отсутствием общего инструментария и механизмов объединения экспертных знаний, большими временными затратами на сбор исходных данных и разработку экспертных оценок при достаточно жестких ограничениях на время принятия решения.
Кроме того, традиционный подход делает основной упор именно на экспертные оценки сложившейся ситуации на объекте управления, по результатам которых и формируется базовая имитационная модель, или, по крайней мере - ее идеологическая основа. В данном случае при существенном расширении предметной области с целью комплексного анализа ситуации уже на первом этапе возникают трудности с оценкой адекватности модели, а также обоснованности ее границ и степени детализации отображения исследуемых процессов.

Наличие отработанной и широкой нормативной базы позволяет принципиально изменить сам подход к формированию моделей развития ЧС. В данной ситуации оказывается гораздо эффективнее формировать базовую модель на основе комплексного анализа имеющихся регламентов и нормативов, а в дальнейшем ее модифицировать с учетом детальной информации о специфике объекта исследования и поступающей оперативной информации о характере развития ситуации. Такой подход дает сразу несколько вполне очевидных преимуществ.

1. Использование технических регламентов и иных нормативов в качестве информационной базы позволяет значительно повысить уровень адекватности разработанной многофакторной модели, поскольку в ее основе лежат достоверные данные об объекте исследования, качество которых прошло многократную проверку в процессе разработки, согласования и утверждения технических регламентов и иных нормативов.

2. Повышается результативность диагностики источников уязвимости на объекте управления при воздействии различного рода угроз, а также точность оценки рисков, характеризующихся относительно низким уровнем вероятности возникновения чрезвычайных ситуаций и ката- 


\section{Тренды и управление - № 2 (2)•2013}

строфическими последствиями в случае реализации рисков.

3. Существенно снижается трудоемкость разработки модели, поскольку она ведется на основе уже согласованных между собой и (как минимум частично) формализованных документов, содержащих обширную, достоверную и, что наиболее важно, значимую информацию с точки зрения целей сценарного исследования.

4. Практически не возникает серьезных проблем согласования и объединения экспертных знаний при разработке базовой модели, поскольку, по сути, наиболее сложные процедуры рассматриваемого типа уже проведены в процессе разработки регламентов и их результаты могут быть прямо использованы при разработке модели.

5. Существенно снижаются затраты времени на диагностический и детальный анализ в процессе разработки многофакторной модели, поскольку необходимые при описании предметной области и наиболее затратные по времени реализации процедуры экспертных оценок проводятся не по всему комплексу проблем, а служат лишь для уточнения необходимых деталей или анализа возникающих непредвиденных обстоятельств в развитии ситуации.

6. При использовании нормативной базы с высоким уровнем детализации появляется возможность проводить исследование имитационной модели на основе количественных оценок и абсолютных шкал, позволяющих осуществлять модельные эксперименты в реальном масштабе времени. Это обеспечивает значительное повышение обоснованности генерируемых сценариев развития ситуации, точности формируемых на их основе прогнозов, а также достоверности оценки эффективности принимаемых управленческих решений.

7. Существенно упрощается наиболее тру- доемкая процедура модификации модели, особенно в случае необходимости ее значительного расширения и охвата смежных предметных областей. Это принципиально позволяет получать комплексные стратегические решения с учетом синергического эффекта в развитии ЧС.

8. Использование нормативно - регламентной базы позволяет заметно упростить решение целого ряда технологических проблем сценарного исследования сложно - структурированных систем, связанных с трудоемкостью процедур оперативного изменения моделей управляемых объектов в условиях ограниченного времени, а также учета динамики изменения наборов рассматриваемых ключевых факторов и параметров, а также структуры их взаимосвязей.

9. И, наконец, пожалуй, самым важным преимуществом широкого использования нормативной базы в процессе сценарного исследования является возможность комплексного подхода к решению задач управления предупреждением и ликвидацией последствий ЧС, позволяющего одновременно рассматривать взаимосвязанные, но принципиально различные по своей природе явления и процессы. Это обеспечивает возможность достаточно эффективного учета в единой модели факторов и угроз террористической, пожарной, радиационной, химической, энергетической, экологической и т.д. безопасности.

\section{Заключение}

Многие страны, и Россия в том числе, сталкиваются с необходимостью ликвидации в кратчайшие сроки последствий крупномасштабных ЧС природного и техногенного характера. Ежегодно в мире случается множество ЧС. Если чрезвычайная ситуация возникает в 
индустриальном районе, крупном городе, она неизбежно ведет к значительным разрушениям и потерям и может унести сотни и тысячи человеческих жизней.

В последние десятилетия в мире наблюдается устойчивая тенденция существенного роста материальных потерь в результате техногенных катастроф и стихийных бедствий, размер которых только в 2011 году достиг рекордного значения в истории, превысив 370 миллиардов долларов США. Одновременно с этим по данным одной из крупнейших в мире страховой компании Swiss Re, число человеческих жертв катастроф различной природы только в 2012 году достигло 14 тысяч человек. Одной из основных причин такого положения помимо ускорившихся в наступившем столетии глобальных климатических изменений и все рельефнее проявляющегося синергетического характера многих техногенных катастроф, является недостаточная готовность систем управления предупреждением и ликвидацией последствий чрезвычайных ситуаций к быстрой, адекватной и эффективной реакции на подобные события.

Существующая тенденция к возрастанию масштабов ЧС и тяжести их последствий обусловливает необходимость своевременной и обоснованной выработки контрмер для их ликвидации и предполагает создание с этой целью соответствующих управленческих структур - систем управления в условиях ЧС. Знание причин возникновения и характера чрезвычайных ситуаций позволяет за счет заблаговременно принятых мер защиты и правильного поведения населения значительно снизить все виды потерь. Поскольку многие катастрофы и стихийные бедствия предотвратить невозможно, то в основу борьбы за уменьшение ущерба и потерь от них должно быть положено своевременное предупреждение населения о грозящем бедствии. Основными направлениями предупреждения чрезвычай- ных ситуаций и потерь от них являются:

- мониторинг окружающей природной среды и состояния объектов народного хозяйства;

- прогнозирование чрезвычайных ситуаций и оценка их риска;

- $\quad$ рациональное размещение производительных сил с точки зрения природной и техногенной безопасности;

- предупреждение аварий и техногенных катастроф путем повышения технологической безопасности производственных процессов и эксплуатационной надежности оборудования;

- подготовка объектов экономики и систем жизнеобеспечения к работе в условиях чрезвычайных ситуаций;

- проведение государственной политики в области защиты населения и территорий от чрезвычайных ситуаций;

- страхование природных и техногенных рисков;

- информирование населения о потенциальных природных и техногенных угрозах на территории проживания.

Анализ развития чрезвычайных ситуаций и принятие оперативных решений осложняются весьма существенной неопределенностью оценок их основных факторов, неоднозначностью в выборе способов их ликвидации, сложностью количественной оценки эффективности принимаемых решений. Руководящим органам даже при значительных материальных ресурсах приходится действовать в условиях острого дефицита времени, ограниченной точности и достоверности информации, что может привести к принятию нерациональных и даже ошибочных решений, а следовательно, и к большим потерям не только материального плана.

В этих условиях существенно возрастает роль теоретических и методологических основ создания и функционирования систем 
управления в условиях чрезвычайных ситуаций, позволяющих существенно повысить их эффективность, а также обеспечить оперативную и бесперебойную работу сил и средств ликвидации последствий ЧС.

Приведенные в настоящей работе методические материалы помогут формализовать и автоматизировать процессы планирования и принятия стратегических и оперативных решений в чрезвычайных ситуациях, а также управления деятельностью по предупреждению ЧС на различных уровнях.

\section{Библиография:}

1. Шульц В.Л., Кульба В.В., Шелков А.Б., Чернов И.В. Методы планирования и управления техногенной безопасностью на основе сценарного подхода // Национальная безопасность/Nota Bene. - 2013, №2 (25).

2. Архипова Н.И., Кульба В.В., Косяченко С.А., Чанхиева Ф.Ю.,Шелков А.Б. Организационное управление. - М.: Изд-во РГГУ, 2007.

3. Информационное обеспечение систем организационного управления (теоретические основы). В 3-х частях. Часть 1. Методологические основы организационного управления. / Под ред. Е.А. Микрина и В.В. Кульбы.-М.: Изд-во физ.-мат. лит., 2011.

4. Модели и методы анализа и синтеза сценариев развития социально-экономических систем: в 2-х кн. / под ред. В.Л. Шульца и В.В. Кульбы. - М.: Наука, 2012.

5. Кульба В.В., Кононов Д.А., Косяченко С.А., Шубин А.Н. Методы формирования сценариев развития социально-экономических систем. - М.: СИНТЕГ, 2004.

6. Кононов Д.А., Кульба В.В., Ковалевский С.С., Косяченко С.А. Формирование сценарных пространств и анализ динамики поведения социально-экономических систем. / Научное издание. - М.: ИПУ РАН, 1999.
7. Шульц В.Л., Кульба В.В., Шелков А.Б., Чернов И.В. Методы сценарного анализа угроз эффективному функционированию систем организационного управления. // Тренды и управление/Nota Bene, 2013, № 1.

8. Владимиров В.А., ВоробьевЮ.Л.,Малинецкий Г.Г., и др. Управление риском. Риск, устойчивое развитие, синергетика. - М.: Наука, 2000.

9. Малинецкий Г.Г., Митин Н.А., Ахромеева Т.С. и др. Когнитивные центры как инструмент управления безопасностью сложных систем // Труды ХХ Международной конференции по проблемам управления безопасностью сложных систем.-М.: ИПУ РАН. 2012.

10. Сергиенко И.В. Математические модели и методы решения задач дискретной оптимизации. Киев: Наук. думка, 1988

11. В.Л. Шульц Сценарный анализ в управлении социальной безопасностью // Национальная безопасность. - 2012. - 6. - С. 4 - 21.

12. В.Л. Шульц, В.В. Кульба, А.Б. Шелков, И.В. Чернов Методы сценарного анализа угроз эффективному функционированию систем организационного управления // Тренды и управление. - 2013. - 1. - С. 6 - 30. DOI: 10.7256/2307-9118.2013.01.2.

13. Ю. П. Липунцов Управление идентификацией в электронном государстве: безопасность данных и неприкосновенность частной жизни // Национальная безопасность. - 2011. - 6. - С. $18-27$.

14. В.В.Цыганов, В. А. Бородин, Г. Б. Шишкин О модернизации системы государственного управления // Национальная безопасность. 2012. - 1. - C. 15 - 26.

15. А. В. Царегородцев, А. К. Качко Один из подходов к управлению информационной безопасностью при разработке информационной инфраструктуры организации // Национальная безопасность. - 2012. - 1. - С. 46 - 59. 


\section{References (transliteration):}

1. Shul'c V.L., Kul'ba V.V., Shelkov A.B., Chernov I.V. Metody planirovaniya i upravleniya tehnogennoy bezopasnost'yu na osnove scenarnogo podhoda // Nacional'naya bezopasnost'/Nota Bene. - 2013, №2 (25).

2. Arhipova N.I., Kul'ba V.V., Kosyachenko S.A., Chanhieva F.Yu., Shelkov A.B. Organizacionnoe upravlenie. - M.: Izd-vo RGGU, 2007.

3. Informacionnoe obespechenie sistem organizacionnogo upravleniya (teoreticheskie osnovy). V 3-h chastyah. Chast' 1. Metodologicheskie osnovy organizacionnogo upravleniya. / Pod red. E.A. Mikrina i V.V. Kul'by.-M.: Izd-vo fiz.-mat. lit., 2011.

4. Modeli i metody analiza i sinteza scenariev razvitiya social'no-ekonomicheskih sistem: v 2-h kn. / pod red. V.L. Shul'ca i V.V. Kul'by. - M.: Nauka, 2012.

5. Kul'ba V.V., Kononov D.A., Kosyachenko S.A., Shubin A.N. Metody formirovaniya scenariev razvitiya social'noekonomicheskih sistem. -M.: SINTEG, 2004.

6. Kononov D.A., Kul'ba V.V., Kovalevskiy S.S., Kosyachenko S.A. Formirovanie scenarnyh prostranstv i analiz dinamiki povedeniya social'no - ekonomicheskih sistem. / Nauchnoe izdanie. - M.: IPU RAN, 1999.

7. Shul'c V.L., Kul'ba V.V., Shelkov A.B., Chernov I.V. Metody scenarnogo analiza ugroz effektivnomu funkcionirovaniyu sistem organizacionnogo upravleniya. // Trendy i upravlenie/Nota Bene, 2013, № 1.
8. Vladimirov V.A., Vorob'ev Yu.L., Malineckiy G.G., i dr. Upravlenie riskom. Risk, ustoychivoe razvitie, sinergetika. - M.: Nauka, 2000.

9. Malineckiy G.G., Mitin N.A., Ahromeeva T.S. i dr. Kognitivnye centry kak instrument upravleniya bezopasnost'yu slozhnyh sistem // Trudy XX Mezhdunarodnoy konferencii po problemam upravleniya bezopasnost'yu slozhnyh sistem.-M.: IPU RAN. 2012.

10. Sergienko I.V. Matematicheskie modeli i metody resheniya zadach diskretnoy optimizacii. Kiev: Nauk. dumka, 1988

11. V.L. Shul'c Scenarnyy analiz v upravlenii social'noy bezopasnost'yu // Nacional'naya bezopasnost'. - 2012. - 6. - C. 4 - 21.

12. V.L. Shul'c, V.V. Kul'ba, A.B. Shelkov, I.V. Chernov Metody scenarnogo analiza ugroz effektivnomu funkcionirovaniyu sistem organizacionnogo upravleniya // Trendy i upravlenie. - 2013. - 1. - C. 6 - 30. DOI: 10.7256/2307-9118.2013.01.2.

13. Yu. P. Lipuncov Upravlenie identifikaciey v elektronnom gosudarstve: bezopasnost' dannyh i neprikosnovennost' chastnoy zhizni // Nacional'naya bezopasnost'. - 2011. - 6. - C. $18-27$.

14. V. V. Cyganov, V. A. Borodin, G. B. Shishkin O modernizacii sistemy gosudarstvennogo upravleniya // Nacional'naya bezopasnost'. 2012. - 1. - C. 15 - 26.

15. A. V. Caregorodcev, A. K. Kachko Odin iz podhodov $\mathrm{k}$ upravleniyu informacionnoy bezopasnost'yu pri razrabotke informacionnoy informacionnoy bezopasnost'yu pri razrabotke informacionnoy infrastruktury organizacii // Nacional'naya bezopasnost'. - 2012. - 1. - C. $46-59$. 